\title{
The Psychological Impact of Diabetic Limb Problems
}

\author{
Bahari R
}

Department of Psychiatry, Faculty of Medicine, Cyberjaya University College of Medical Sciences, No 3410 Jalan Teknokrat 3, Cyber 4, 63000 Cyberjaya, Selangor.

Hospital Pakar An Nur Hasanah, 14 \& 16, Medan Pusat Bandar 1, Seksyen 9, 43650 Bandar Baru Bangi, Selangor.

\begin{abstract}
Limb problem is a common complication for patients with diabetes. While the impact of diabetic limb problems on physical health is well known, the psychological impact of the condition is still largely unknown. People with diabetes with or without foot ulcers frequently suffer from major depressive disorder, and this is made worse by the presence of limb complications. Furthermore, depression itself can have negative consequences on the person's diabetes. Hence, in caring for people with diabetic foot problem, the psychological sequelae must not be overlooked but managed accordingly.
\end{abstract}

Keywords: diabetes; diabetic limb problem; psychological impact; depression; anxiety

\section{INTRODUCTION}

Diabetes Mellitus is a common illness worldwide. In 2011, an estimated $8.3 \%$ of adults globally are affected by the condition and this number is expected to raise by $50.7 \%$ by the year $2030 .{ }^{1}$ In Malaysia, the prevalence in 2013 was estimated at $22.6 \%$, double the rate reported in $2006 .{ }^{2}$ The condition also places a lot of burden on the health system and economy. Hospitalisation, medication and complications of the condition results in excessive economic costs. ${ }^{3}$

One of the most frequent and disabling complication of diabetes is a group of condition known as diabetic limb problems. Diabetic foot problems, such as ulcers, infection, gangrene often results from peripheral neuropathy and vascular diseases. ${ }^{4}$ In itself, foot ulcers are disabling and lead to repeated hospitalisations, but amputation is the more feared outcome of the condition. ${ }^{5}$ Diabetic hand problems, in contrast, do not get as much exposure as its counterpart of the foot, but are nevertheless common and important. They include the diabetic hand syndrome, which is characterised by three conditions; diabetic cheiroarthropathy or limited joint movements, flexor tenosynovitis or trigger finger and Dupuytern's Disease. ${ }^{6}$ Another common complication of the hands

Corresponding author:

Rafidah Bahari

Department of Psychiatry, Faculty of Medicine, Cyberjaya University College of Medical Sciences, No 3410 Jalan Teknokrat 3, Cyber 4, 63000 Cyberjaya, Selangor.

Phone: +60383137104. Fax: +60383137001.

E-mail: rafidahbahari@cybermed.edu.my. is Carpal Tunnel Syndrome, which occur in $14-60 \%$ of diabetic patients. ${ }^{7}$

Diabetic patients often demonstrate psychiatric vulnerability at diagnosis and when complications are detected. ${ }^{8}$ The relationship between mental illness and diabetes is complicated, bidirectional and not fully understood. ${ }^{9}$ The most prevalent mental illness associated with diabetes is depression, followed closely by anxiety disorders. ${ }^{10}$ Type 2 diabetes mellitus is especially widespread among patients with schizophrenia and bipolar disorder, and i partly explained by the long-term use of antipsychotic medications. ${ }^{11}$ Numerous research have been done to study the psychological and social consequences of diabetes. However, only a handful of studied looked at the psychological impact of diabetic limb problems.

\section{PSYCHOLOGICAL IMPACT OF DIABETIC LIMB PROBLEMS}

While the physical impacts of diabetic limb problems are well known, the impact on the psychological well-being of the sufferers have not been well studied. Most research on psychological consequences of diabetic limb problems were done on diabetic foot and some on amputations. Studies on diabetic hands were extremely limited. From these studies, it is found that the most frequent psychological manifestation were again, depression and anxiety disorders. The psychological impact of the most common diabetic limb problems are elaborated below.

\section{Psychosocial effect of diabetic foot ulcers}

Lower limb complications of diabetes costed US taxpayers USD 10. ${ }^{9}$ billion in 2001 and Malaysian government almost USD 84 million in 2014. 5,12 The costs were mainly made up of admissions, medications, investigations and surgical interventions. These are direct costs of diabetes-related foot complications. In addition, there are also indirect costs arising from 
loss of productivity and reduction in quality of life. The social impact of diabetic foot ulcers (DFU) should not be overlooked as they could lead to psychological conditions. In a study involving 290 participants with DFU, Bajwa et. al. ${ }^{10}$ found that $12 \%$ had to leave their employment due to reduced mobility, about $17 \%$ felt they were a burden to their family and $7.5 \%$ were so distressed by it that they admitted to have lost hope in life. This is supported by Fejfarová et al. ${ }^{13}$ who found that patients with diabetes had lower quality of life and almost half were on disability pensions. They also found that DFU limits social contacts due to their reduction in mobility, which also adds to their distress and in turn affecting their mental health.

\section{Depression}

Depression is the most important mental illness affecting patients with diabetes, ${ }^{14}$ and the risk of developing the condition is higher in patients with diabetes-related complications. ${ }^{15,16}$ It is found that up to a third of patients with DFU have depressive disorders. ${ }^{17}$ Those aged less than 45 at first presentation of DFU, had chronic DFU, had larger baseline ulcers and poor social support are particularly at risk. ${ }^{10,18}$ Having co-morbid depression and diabetes are detrimental to patients. Depression worsens diabetes through a variety of ways which may not be easy to explain. It can influence diabetes and its complications through biological pathways (alteration in glucose transport and hormonal abnormalities) and negative health behaviours (inactivity, smoking, poor diet and poor self-care). ${ }^{11}$ Depression is also the most important determinant of quality of life in patients with diabetes. ${ }^{19}$ Ultimately, depressive disorders increases the risk of death in patients with DFU, with almost threefold increase at 18 months. ${ }^{18}$

On the other hand, depression may precede the diagnosis diabetes indicating that it in itself is a risk factor for diabetes. ${ }^{20}$ Fortunately, studies have shown that this increase the risk is slight. ${ }^{16}$ Heavy smoking, lack of physical activity, excessive alcohol drinking are all lifestyle factors associated with depression which increases risk of developing diabetes. ${ }^{21}$ The biological factors as stated above also contributes. It was also found that depression may increase the risk of developing DFU through negative effects on patients' compliance to treatment, which in turn results in poor glycaemic control and subsequently wound healing. ${ }^{13}$ Antidepressants especially monoamine oxidase inhibitors (MAOI) and tricyclic antidepressants (TCA) can worsen glycaemic control hence worsening foot ulcers. ${ }^{10}$ Poor self-care especially in looking after their foot may aggravate existing DFU and the vicious cycle continues. ${ }^{22}$

\section{Anxiety Disorders}

Those with diabetes mellitus are more likely to have anxiety disorders than those without. ${ }^{20}$ Almost $40 \%$ of people with diabetes have elevated anxiety levels and about $14 \%$ fulfils the diagnosis of generalised anxiety disorders (GAD). ${ }^{9}$ Anxiety is positively correlated with diabetes education difficulty, complications of diabetes and age. ${ }^{23}$ Wound healing appears to be important predictor to anxiety in which those with recurrent non-healing foot ulcers present with more anxiety symptoms than those with healed ulcers. ${ }^{10}$

Anxiety can also contribute to worsening of diabetes and its complications. Fears of injecting insulin especially if the person have specific phobia to needles makes self-injecting and blood glucose monitoring difficult. ${ }^{24}$ In anxious patient with DFU, high-levels of stress-induced glucocorticoids suppresses interleukin-1 B which plays a very important part in wound healing. ${ }^{10}$ Similar to depression, there is a vicious cycle with nonhealing ulcers and anxiety, reinforcing the complex relationship between mental illness and diabetes.

\section{Adjustment Disorders}

Adjustment disorders can present similar to mood or anxiety disorders but the symptoms are self limiting. ${ }^{25}$ They often present at diagnosis or when foot ulcers are present, but with time symptoms spontaneously diminish to disappear or become less troublesome after one month. Patients newly diagnosed with diabetes have a lot of lifestyle adjustments to make and may become distressed while they get used to the new arrangements. Some reported being ashamed of injecting insulin at work or school and having difficulty to control their blood glucose contributing to the above condition. ${ }^{24}$ Patients with unhealed foot ulcers also reported significantly lower scores on psychosocial adjustment to illness scale than diabetic patients with healed ulcers. ${ }^{26}$ In addition, those with DFU perceived their physical health as poorer than those with diabetes but without DFU. ${ }^{27}$ As long as foot ulcers are present, the distress of living with them, the poor perceived physical health, the pain, the reduction in mobility and fears of ulcers not healing leading to adjustment disorders.

\section{Mental health issues associated with amputation}

Amputation is the most dreaded consequence of diabetic limb problem and understandably results in various manifestations of psychological distress. Amputees were found to have lower quality of life due to unemployment and social isolation and also described worse stress readaptation compared to people with diabetes but without amputations. ${ }^{13}$ Bhuvaneswar et al. ${ }^{22}$ reported that patients who had to undergo amputation may experience grief, adjustment disorder, depression, anxiety and post traumatic stress disorder (PTSD). Upon discovering that amputation is required, patients may go through alternating periods of anxiety and depression directed towards the perceived negative attitudes of people towards amputees. Following the amputation, grief or adjustment disorder may develop. Anxiety and depression 
are also considerable in people who had undergone amputations, but whether it is due to phantom limb pain remains inconclusive. ${ }^{28}$ Factors which are known to increase risk of developing major depressive disorder are young age at amputation, anxious personality and poor coping skills. ${ }^{22}$

PTSD following surgical amputation of limbs are relatively rare. Less than $5 \%$ of people who had undergone amputation due to chronic illness like diabetes develop PTSD. ${ }^{10}$ The condition is more common in amputations due to combat or accidental injury, in hand and digit amputations, following difficult surgeries as well as in those with extensive immobility. ${ }^{22}$

Interestingly, it is not all doom and gloom for amputees. Price ${ }^{29}$ reported that diabetic patients with foot ulcers who had undergone amputations had better quality of life, more satisfied with their personal life, had more positive attitudes toward foot care and, especially if they are fitted with prostheses are more mobile than those with DFU without amputation. The author concluded that although amputations are seen as drastic by both patients and doctors, they do offer better mobility and better quality of life than those with recurrent non-healing ulcers. Hence, amputation is not only necessary in preventing spread of infection in but may also improve quality of life in patients with DFU.

\section{MANAGEMENT}

\section{Assessment}

The detrimental effect of mental illness on diabetes and diabetic limb problems have been discussed above and it is important that psychiatric conditions in people with diabetes are recognised and treated. However, these patients rarely complain of and present to their doctors with psychiatric symptoms. In a study involving 253 people with diabetic foot ulcers, approximately $32 \%$ fulfilled the diagnosis of depressive disorder, but only $12 \%$ of those with a positive diagnosis received antidepressant treatment. ${ }^{17}$ Hence, there is a need to screen patients for mental illness especially at times where patients are most psychologically vulnerable.

Numerous screening tools have been found to be useful in this particular group of patients. Tools such as Beck Depression Inventory (BDI), Centres for Epidemiologic Studies Depression Scale (CES-D) as well as Hospital Anxiety and Depression Scale (HADS) have all been validated for use in the diabetic population. ${ }^{30}$ Specific questionnaires to assess diabetes-specific difficulties may also be helpful to identify fears related to self-injecting, monitoring and others. These can be done through the application of screening tools such as the Diabetes Distress Scale, Diabetes Quality of Life Questionnaire and Hypoglycaemia Fear Survey. ${ }^{11}$

\section{Diabetes patient education}

The American Diabetes Association recommends that every patient receive diabetes self-management education (DSME) upon diagnosis. ${ }^{8}$ Psychosocial issues is an integral part of the DMSE highlighting the importance of mental health in people with diabetes. They also reported that patients who received DSME had better knowledge and self-care, better glycaemic control, improved quality of life, more positive coping and lower cost of care. Educational programmes must take into account cultural and societal sensitivities and are tailored to patients' specific needs. ${ }^{5}$

\section{Multidisciplinary management}

Given the complexity of diabetic limb problems, the best approach to care is multidisciplinary. Especially when the patient with diabetic limb problem has comorbid mental illness, collaborative care between physicians, surgeons, psychiatrists, podiatrists, pharmacists, nurse, family physicians and other health care provider is necessary to effectively manage the patient. ${ }^{20}$

Anti-depressants are typically used in the treatment of anxiety and depressive disorders. Serotonin Selective Reuptake Inhibitors (SSRI) are the first line medications and have been found to be effective in reducing symptoms and preventing relapse in diabetic patients. ${ }^{31}$ In addition, they also help to a small extent improve glycaemic control, possibly through improving patients' motivation to care for their diabetes and feet better. However, tricyclic antidepressants such as nortriptyline, while are effective to reduce symptoms of depression, may worsen glycaemic care. ${ }^{31}$ Also, in depression which are more resistant to treatment, the use of atypical antipsychotics are becoming more popular with increasing evidence for them. Again, atypical may worsen glycaemic control along with predisposing patients to the triad of weight gain, hypercholesterolaemia and hypertension. ${ }^{14}$ Hence, the decision to commence antidepressant needs to be made together with the patient's psychiatrists.

Psychosocial interventions may also benefit patients with diabetic limb problems. Nutritional advice and exercise are beneficial, not only to control weight but also for promoting mental wellness. Strong support from family and friends can reduce or prevent anxiety in patients with DFU and improve their outcome. ${ }^{11}$ Incorporating spiritual interventions can be useful in promoting acceptance and reduce psychiatric symptoms in DFU and especially following amputations. ${ }^{22}$ Many interventions have been tried and appear helpful in reducing stress and anxiety as well as improvement in glycaemic control such as biofeedback-assisted relaxation training and mindfulness-based stress management. ${ }^{11}$ In terms of increasing compliance to treatment and coping with DFU, supportive therapy, motivational interviewing and behavioural therapy may be useful. ${ }^{9}$ Cognitive 
Behavioural Therapy (CBT) is recommended for the treatment of anxiety and major depressive disorders. They are effective in reducing symptoms of mental illness but evidence for blood glucose control are conflicting. There is evidence that psychotherapy in combination with diabetes self-management is the best treatment than either interventions alone in terms of depressive symptoms and glycaemic control. ${ }^{32}$ The same strategy should also be applicable to other psychiatric conditions such as anxiety and adjustment disorder.

In summary, the aim of treatment in patients with diabetic foot ulcer is still good control of blood sugar levels and proper foot care. The presence of mental health issues makes achieving the above objective difficult. Hence, it is important to prevent them in the first place by addressing psychosocial issues during patient education and follow-up. In vulnerable patients, screening and, if necessary, prompt referral is essential so that mental illness in this population can be managed before they deteriorate. In patients with diabetic limb problems and diagnosed mental illness, the holistic approach using bio-psycho-social and spiritual techniques are the most effective and contribute to the most improvement of outcome.

\section{CONCLUSION}

Psychological consequences of diabetic limb problem may be more widespread and serious than it is thought. Given the complicated and bidirectional relationship between diabetes and mental illness, their identification and proper management are essential. Multidisciplinary care is the key to successful management of mental health issues in diabetes and diabetic limb problems. Patient education on diabetes, wound care and increase of support prior to amputations are helpful to reduce distress and may even have a role in preventing serious mental illness.

\section{REFERENCES}

1. Whiting DR, Guariguata L, Weil C, Shaw J. IDF diabetes atlas: global estimates of the prevalence of diabetes for 2011 and 2030. Diabetes Res Clin Pract. 2011;94(3):311-321. doi:10.1016/j.diabres.2011.10.029.

2. Wan Nazaimoon WM, Md Isa SH, Wan Mohamad WB, et al. Prevalence of diabetes in Malaysia and usefulness of $\mathrm{HbA} 1 \mathrm{C}$ as a diagnostic criterion. Diabet Med. 2013;30(7):825-828. doi:10.1111/ dme.12161.

3. Atlantis E. Excess burden of type 1 and type 2 diabetes due to psychopathology. J Affect Disord. 2012;142 Suppl:S36-S41. doi:10.1016/ S0165-0327(12)70007-1.

4. Korzon-burakowska A, Dziemidok P. Diabetic foot - the need for comprehensive multidisciplinary approach. Ann Agric Environ Med. 2011;18(2):314- 317.

5. Boulton A. An Integrated Health Care Approach is Needed: The Global Burden of Diabetic Foot Disease. Diabet Microvasc Complicat Today. 2006;
(February):23-26.

6. Papanas N, Maltezos E. The diabetic hand: a forgotten complication? J Diabetes

Complications. 2010;24(3):154-162. doi:10.1016/ j.jdiacomp.2008.12.009.

7. Al-Matubsi HY, Hamdan F, Alhanbali $\mathrm{O}$ a, Oriquat $\mathrm{G}$ a, Salim M. Diabetic hand syndromes as a clinical and diagnostic tool for diabetes mellitus patients. Diabetes Res Clin Pract. 2011;94(2): 225-229. doi:10.1016/j.diabres.2011.07.012.

8. Association AD. Standards of medical care in diabetes--2010. Diabetes Care. 2010;33 Suppl 1:S11-S61. doi:10.2337/dc10-S011.

9. Britneff E, Winkley K. The role of psychological interventions for people with diabetes and mental health issues. J Diabetes Nurs. 2013;17(8): 305-310.

10. Bajwa SJ, Gagandeep K, Harbandna S, Neeru B, Pal A, Rajesh K. Psychosocial, psychiatric, and clinical implications of diabetic foot ulceration: A prospective analysis. J Soc Heal Diabetes. 2015;3(2):89. doi:10.4103/

2321-0656.152805.

11. Gonzalez JS, Esbitt SA, Schneider HE, Osborne PJ, Kupperman EG. Psychological Co-Morbidities of Physical Illness: A Behavioural Medicine Perspective. (Pagoto S, ed.). New York, NY: Springer New York; 2011:73-122. doi:10.1007/978-1-4419-0029-6.

12. Lam A, Zaim M, Helmy H, Ramdhan I. Economic Impact of Managing Acute Diabetic Foot Infection in a Tertiary Hospital in Malaysia. Malaysian Orthop J. 2014;8(1):46-49. http:// www.ncbi.nlm.nih.gov/pmc/articles/ PMC4093540/. Accessed October 30, 2015.

13. Fejfarová $V$, Jirkovská $A$, Dragomirecká $E$, et al. Does the diabetic foot have a significant impact on selected psychological or social characteristics of patients with diabetes mellitus? J Diabetes Res. 2014;2014:371938. doi:10.1155/2014/371938.

14. Rustad JK, Musselman DL, Nemeroff CB. The relationship of depression and diabetes: pathophysiological and treatment implications. Psychoneuroendocrinology. 2011;36(9): 1276-1286. doi:10.1016/j.psyneuen.2011.03.005.

15. Nouwen a, Winkley K, Twisk J, et al. Type 2 diabetes mellitus as a risk factor for the onset of depression: a systematic review and meta-analysis. Diabetologia. 2010;53(12): 2480-2486. doi:10.1007/s00125-010-1874-x.

16. Roy T, Lloyd CE. Epidemiology of depression and diabetes: a systematic review. J Affect Disord. 2012;142 Suppl:S8-S21. doi:10.1016/ S0165-0327(12)70004-6.

17. Ismail K, Winkley K, Stahl D, Chalder T, Edmonds M. A Cohort Study of People With Diabetes and Their First Foot Ulcer. Diabetes Care. 2007;30:1473-1479. doi:10.2337/ dc06-2313.Abbreviations.

18. Winkley K, Sallis $\mathrm{H}$, Kariyawasam $\mathrm{D}$, et al. Five-year follow-up of a cohort of people with their first diabetic foot ulcer: the 
persistent effect of depression on mortality. Diabetologia. 2012;55(2):303-310. doi:10.1007/ s00125-011-2359-2.

19. Wexler DJ, Grant RW, Wittenberg E, et al. Correlates of health-related quality of life in type 2 diabetes. Diabetologia. 2006;49(7): 1489-1497. doi:10.1007/s00125-006-0249-9.

20. Egede LE, Ellis C. Diabetes and depression: Global perspectives. Diabetes Res Clin Pract. 2010;87(3): 302-312. doi:10.1016/j. diabres.2010.01.024.

21. Atlantis E, Vogelzangs N, Cashman K, Penninx BJWH. Common mental disorders associated with 2-year diabetes incidence: the Netherlands Study of Depression and Anxiety (NESDA). J Affect Disord. 2012; 142 Suppl:S30-S35. doi:10.1016/S0165-0327(12) 70006-X.

22. Bhuvaneswar CG, Epstein LA, Stern TA. Reactions to Amputation: Recognition and Treatment. Prim Care Companion J Clin Psychiatry. 2007;9(4):303-308.

23. Wu S-FV, Huang Y-C, Liang S-Y, Wang T-J, Lee $\mathrm{M}-\mathrm{C}$, Tung $\mathrm{H}-\mathrm{H}$. Relationships among depression, anxiety, self-care behaviour and diabetes education difficulties in patients with type-2 diabetes: A cross-sectional questionnaire survey. Int J Nurs Stud. 2011; 48(11):1376-1383.doi:10.1016/j. ijnurstu.2011.04.008.

24. Dalvi M, Feher M, Caglar E, Catalan J. Liaison psychiatrist in a specialist diabetes centre. Psychiatr Bull. 2008;32(12):461-463. doi:10.1192/pb.bp.107.018358.

25. American Psychiatric Association. Diagnostic and Statistical Manual of Mental Disorders. 5th Ed (DSM 5). Fifth Edit. American Psychiatric Association; 2013:991.

26. Goodridge D, Trepman E, Embil JM. HealthRelated Quality of Life in Diabetic Patients With Foot Ulcers: Literature Review. J Wound, Ostomy Cont Nurs. 2005;32(6):368-377.

27. Iversen MM, Midthjell K, Tell GS, et al. The association between history of diabetic foot ulcer, perceived health and psychological distress: the Nord-Trøndelag Health Study. BMC Endocr Disord. 2009;9(18). doi:10.1186/14726823-9-18.

28. Kazemi H, Ghassemi S, Fereshtehnejad SM, Amini A. Anxiety and Depression in Patients with Amputated Limbs Suffering from Phantom Pain : A Comparative Study with Non Phantom Chronic Pain. 2013;4(2).

29. Price P. The diabetic foot: quality of life. Clin Infect Dis. 2004;39 Suppl 2:S129-S131. doi:10.1086/383274.

30. Petrak F, Baumeister H, Skinner T. Depression and diabetes: treatment and health-care delivery. Lancet Diabetes Endocrinol. 2015:25-38. http://www.sciencedirect.com/science/ article/ pii/S2213858715000455. Accessed November 1, 2015.
31. Markowitz SM, Gonzalez JS, Wilkinson JL, Safren $S$ a. A review of treating depression in diabetes: emerging findings. Psychosomatics. 2011;52(1): 1-18. doi:10.1016/j.psym.2010.11.007.

32. Van der Feltz-Cornelis CM, Nuyen J, Stoop C, et al. Effect of interventions for major depressive disorder and significant depressive symptoms in patients with diabetes mellitus: a systematic review and meta-analysis. Gen Hosp Psychiatry. 2010;32(4):380-395. doi:10.1016/j. genhosppsych.2010.03.011. 
